\title{
p53-independent role of MYC mutant T58A in the proliferation and apoptosis of breast cancer cells
}

\author{
DANDAN JIANG $^{1}$, YUHUA SONG ${ }^{1}$, WEIHONG CAO ${ }^{1}$, XINGANG WANG $^{1}$, \\ DANNI JIANG ${ }^{2}$, ZHIDONG LV ${ }^{1}$, ZHAOCHUAN YANG ${ }^{1}$ and FUNIAN LI ${ }^{1}$ \\ ${ }^{1}$ Breast Center; ${ }^{2}$ Imaging Department, The Affiliated Hospital of Qingdao University, Qingdao, Shandong 266003, P.R. China
}

Received February 28, 2018; Accepted September 24, 2018

DOI: $10.3892 / \mathrm{ol} .2018 .9688$

\begin{abstract}
Myc proto-oncogene (MYC) is an oncoprotein that promotes proliferation and apoptosis. MYC mutations frequently disrupt the apoptotic processes during tumorigenesis. In the present study, the effects of the MYC point mutation T58A on the progression of a cellular tumor antigen p53 (p53) ${ }^{-/-}$human breast cancer cell line was analyzed, and the mechanism of p53-independent MYC-induced apoptosis was investigated. HCC1937 cells were transfected with mutant (T58A) or wild-type (WT) MYC using lentiviral vectors. The proliferation of transfected cells was evaluated by colony formation and MTT assays, and apoptosis was analyzed by flow cytometry and terminal deoxynucleotidyl transferase-mediated dUTP nick end labeling assays. WT MYC was transfected into HCC1937 cells exhibiting p14/p21 silencing through lentivirus-mediated RNA interference. The expression levels of Bim were detected by reverse transcription-quantitative polymerase chain reaction and western blot analyses. Mutant MYC proteins retained the ability to stimulate the proliferation of HCC1937 cells, although they were defective at promoting apoptosis due to a failure to induce the Bcl-2 homology 3 domain-only protein Bim. When p14 was silenced, the effects of mutant MYC on proliferation and apoptosis were weakened. When p21 was silenced, the effects of mutant MYC were strengthened. Breast cancer-derived T58A MYC mutations are unable to activate Bim due to their failure to regulate $\mathrm{p} 14 / \mathrm{p} 21$. It was concluded that mutant MYC was more effective compared with WT MYC at promoting the progression of breast cancer.
\end{abstract}

Correspondence to: Dr Funian Li, Breast Center, The Affiliated Hospital of Qingdao University, 59 Haier Road, Qingdao, Shandong 266003, P.R. China

E-mail: dr_lifunian@126.com

Key words: breast cancer, Myc proto-oncogene mutant, Bim, cellular tumor antigen p53-independent, p14, p21

\section{Introduction}

Initially discovered in avian genomes, MYC proto-oncogene (MYC) is a key regulator of cell growth, proliferation, metabolism, differentiation and apoptosis (1), and a potent activator of tumorigenesis. MYC is dysregulated in various cancer types (1-3) and serves a critical role in breast tumorigenesis and cancer progression (4-6). Dysregulation of MYC in breast cancer involves multiple mechanisms, including gene amplification, transcriptional regulation, and mRNA and protein stabilization. Previous studies demonstrated that the MYC T58A point mutation impaired the apoptotic potential of MYC in rodent cells (7-9), and a study reported that this mutation reduced the occurrence of MYC-induced apoptosis in human mammary epithelial cells (10). However, the physiological functions of MYC and the consequences of its dysfunction in breast cancer cells remain obscure.

Bim is a Bcl-2 homology 3 domain (BH3)-only protein that is involved in stimulus-induced, cellular tumor antigen p53 (p53)-independent apoptosis $(11,12)$. MYC was reported to bind to the Bim promoter and promote Bim transcription $(13,14)$. Furthermore, dysregulated MYC expression sensitized cells to apoptosis via a Bim-dependent mechanism (11). Although mutant MYC was unable to induce Bim expression, it activated the p53 pathway to a similar extent as wild-type (WT) MYC (9). This result may help explain why tumors with mutant MYC are less prone to apoptosis, and why mutant MYC is more oncogenic compared with WT MYC (9). Previously, it was demonstrated that tumor-derived MYC mutants are relevant to breast cancer pathology as they are unable to upregulate Bim, which induces p53-independent apoptosis (15). However, the mechanisms underlying the observation that MYC mutants are unable to upregulate Bim are unknown.

The principal model for the p53-independent mechanism of apoptosis is that the tumor suppressor p14, which binds to MYC directly and blocks the transcription of MYC canonical target genes, also inhibits MYC-induced hyperproliferation and transformation $(16,17)$. Despite its inhibition of canonical MYC activity, p14 has been reported to be essential for MYC to induce p53-independent apoptosis in mouse embryonic fibroblasts $(17,18)$. A subsequent study demonstrated that E2F proteins serve a direct role in the transcriptional regulation of p14 (19). Upregulated Bim expression levels in prostate and breast cancer cells are dependent on E2F, and E2F silencing 
leads to the loss of Bim expression (20). Thus, WT MYC appears to upregulate p14 in order to promote Bim-induced apoptosis. However, the mechanisms by which MYC mutants regulate p14-induced p53-independent apoptosis have not been fully clarified.

The cyclin-dependent kinase inhibitor protein p21 regulates cell cycle progression at the G1 phase, mediates cell proliferation, differentiation, senescence and apoptosis, and may influence transcription in a p53-dependent or independent manner (21). Substantial data from biochemical and genetic studies indicate that p21 acts as a master effector of multiple tumor suppressor pathways to promote antiproliferative activities that are independent of the classical p53 tumor suppressor pathway. Furthermore, p21 suppresses the induction of proapoptotic genes by MYC and E2F1 through direct binding and inhibition of their transactivation functions (22). G1/S arrest induced by overexpression of p21 was reported to involve suppression of Bim (23). Although a number of studies indicated a proapoptotic role for $\mathrm{p} 21$, these studies only demonstrated that apoptosis occurred concurrently with p21 induction, without determining whether p21 was required for the induction of apoptosis.

Apoptosis in response to dysregulated MYC is an important failsafe mechanism that is essential in preventing the proliferation of tumorigenic cells (24). Apoptosis induced by oncogenic MYC occurs through p53-dependent and independent mechanisms that are not well understood $(9,25)$. The p53 gene is mutated in $~ 50 \%$ of human tumors, and the loss of p53 antitumor activity is associated with defects in cell cycle arrest and apoptosis (26). It has been proposed that the loss of p53-initiated DNA repair processes underpins the high cancer susceptibility observed in p53-deficient mice (27). DNA damage elicited by chemotherapeutic drugs may induce apoptosis in the absence of p53 (28). It is apparent that various p53-independent apoptotic mechanisms may be used to induce apoptosis in p53-initiated cancer cells. It is therefore important to further clarify the precise molecular mechanism of p53-independent apoptosis. It appears likely that DNA lesions may activate $\mathrm{BH} 3$-only proteins in a p53-independent manner (29). A recent study reported that the loss of Bim strongly correlates with the loss of p53 in lymphomagenesis (11). Thus, the effects of the MYC point mutation T58A on the progression of breast cancer with p53 loss were analyzed, and the mechanism of p53-independent MYC-induced apoptosis was investigated.

MYC dysregulation contributes to the initiation and progression of breast cancer and is associated with poor outcomes, particularly in the basal-like cancer subtype. Thus, targeting MYC-regulated pathways may provide a promising therapeutic strategy for breast cancer.

\section{Materials and methods}

Cells and reagents. Human breast cancer cells (HCC1937; China Center for Type Culture Collection, Chinese Academy of Sciences, Shanghai, China) were cultured in RPMI-1640 medium (HyClone; GE Healthcare Life Sciences, Logan, UT, USA) and $10 \%$ fetal bovine serum (FBS; Hyclone; GE Healthcare Life Sciences). The WT MYC-green fluorescent protein (GFP)-lentivirus and T58A-GFP-lentivirus vectors were constructed previously (30). The p21-small interfering (si)RNA-red fluorescent protein (RFP)-lentivirus and p14-siRNA-RFP-lentivirus vectors were constructed by Shanghai Biological Engineering Co., Ltd. (Shanghai, China). The terminal deoxynucleotidyl transferase-mediated dUTP nick end labeling (TUNEL) assay kit was obtained from Beyotime Institute of Biotechnology (Haimen, China). Antibodies against MYC, p21, p14 and GAPDH were purchased from Abcam (Cambridge, UK). Purified rabbit anti-Bim was from BD Pharmingen (BD Biosciences, Franklin Lakes, NJ, USA).

T58A and WT MYC transfection and p21 and p14 interference of HCC1937 cells. Control lenti-GFP/neo virus, lenti-T58A-GFP/neo, and lenti-WT MYC-GFP/neo particles were previously constructed and packaged (30). The titer was measured using the method described by Meng et al (30). HCC1937 cells were maintained in RPMI-1640 medium supplemented with 10\% FBS and plated into six-well plates at $1 \times 10^{5}$ cells/well overnight. Cells at $50 \%$ confluence were infected with lenti-GFP/neo, lenti-T58A-GFP/neo, lenti-WT MYC-GFP/neo particles, and with $\mathrm{p} 21$ - or $\mathrm{p}$ 14-siRNA-RFP-lentivirus vectors at a multiplicity of infection of 6. Cells were cultured in RPMI-1640 medium supplemented with $10 \% \mathrm{FBS}$ at $37^{\circ} \mathrm{C}$ in $5 \% \mathrm{CO}_{2}$ for 2 days. Transfection efficiencies were determined by assessing GFP expression using a Leica DMI 4000B (Leica Microsystems $\mathrm{GmbH}$, Wetzlar, Germany) fluorescence microscope at magnification, $\mathrm{x} 100$.

NeoMYCin G418 (400 mg/l; Thermo Fisher Scientific, Inc., Waltham, MA, USA) was added to the medium to select the stably transfected cells. NeoMYCin-resistant colonies were picked up 2 weeks post-transfection. Stably transfected cells were maintained in medium containing $400 \mathrm{mg} / 1$ neoMYCin.

RNA extraction and reverse transcription-semi-quantitative polymerase chain reaction ( $R T-s q P C R)$ analysis. Total RNA was isolated from the cells using TRIzol reagent (Thermo Fisher Scientific, Inc.). The first strand of cDNA was synthesized by reverse transcription of $2 \mu \mathrm{g}$ total RNA using an Advantage RT-for-PCR kit (Takara Biotechnology Co., Ltd., Dalian, China). The temperature protocol for reverse transcription was as follows: $72^{\circ} \mathrm{C}$ for $2 \mathrm{~min}, 42^{\circ} \mathrm{C}$ for $1 \mathrm{~h}$ and $94^{\circ} \mathrm{C}$ for $5 \mathrm{~min}$. A $0.6 \mu \mathrm{l}$ aliquot of the RT reaction mixture was used for subsequent sqPCR analysis. The sequences of the forward and reverse primer pairs (designed and synthesized by Shanghai Sangon Biological Engineering Technology Co., Ltd., Shanghai, China) were as follows: MYC sense, 5'-GATTCTCTGCTCTCCTCGAC-3' and antisense, 5'-TCCAGACTCTGACCTTTTGC-3'; p21 sense, 5'-ACTGTGATGCGCTAATGGC-3' and antisense, 5'-ATG GTCTTCCTCTGCTGTCC-3'; p14 sense, 5'-CACCGGAAT CCTGGACCAG-3' and antisense, 5'-GCAGTTCGAATC TGCACCGT-3'; Bim sense, 5'-AGATCCCCGCTTTTCATC TT-3' and antisense, 5'-AGGACTTGGGGTTTGTGTTG-3'; and GAPDH sense, 5'-CTGCACCACCAACTGCTTAG-3' and antisense, 5'-TGAAGTCAGAGGAGACCACC-3'. Each $20 \mu \mathrm{l}$ volume of PCR mixture contained $10 \mu \mathrm{l}$ premixed Taq polymerase (Takara Biotechnology Co., Ltd.), $0.5 \mu$ l forward primer, $0.5 \mu \mathrm{l}$ reverse primer and $8.4 \mu \mathrm{l}$ double-distilled water. 
The PCR thermocycling conditions were as follows: $94^{\circ} \mathrm{C}$ for $3 \mathrm{~min}$, followed by 30 cycles of $94^{\circ} \mathrm{C}$ for $45 \mathrm{sec}, 60^{\circ} \mathrm{C}$ for $45 \mathrm{sec}$ and $70^{\circ} \mathrm{C}$ for $2 \mathrm{~min}$, and a final extension step at $72^{\circ} \mathrm{C}$ for $7 \mathrm{~min}$. A 2- $\mu 1$ aliquot of $\mathrm{PCR}$ product was analyzed by electrophoresis on a $2 \%$ agarose gel containing ethidium bromide, visualized under UV light, and quantified using ImageJ (version 2.1.4.7; https://imagej.nih.gov/ij/).

Western blot analysis. Cells were harvested using radioimmunoprecipitation assay lysis buffer (Sigma-Aldrich; Merck KGaA, Darmstadt, Germany). The protein concentration of each cell extract was measured using a bovine serum albumin protein assay kit (Bio-Rad Laboratories Inc., Hercules, CA, USA). Proteins were separated by SDS-PAGE on a $10 \%$ gel $(30 \mu \mathrm{g} /$ lane). Proteins were transferred to Hybond-P polyvinylidene difluoride membranes (GE Healthcare Life Sciences), which were blocked with $5 \%$ skimmed milk in Tris-buffered saline containing $0.1 \%$ Tween-20 for $60 \mathrm{~min}$ at room temperature. The membranes were incubated overnight at $4^{\circ} \mathrm{C}$ with FLAG-conjugated primary antibodies, including anti-p21, anti-p14, anti-MYC and anti- $\beta$-actin (cat. nos. sc-53393, sc-71808, sc-70469 and sc-58673, respectively; all 1:200 dilution; Santa Cruz Biotechnology, Inc., Dallas, TX, USA), and anti-Bim (cat. no. 559685; 1:200 dilution; BD Pharmingen), followed by incubation at room temperature for $1 \mathrm{~h}$ with an anti-FLAG horseradish peroxidase (HRP)-conjugated monoclonal antibody (cat. no. A8592; 1:5,000 dilution; Sigma-Aldrich; Merck KGaA) in $20 \mathrm{mM}$ TBS with Tween-20. Immunoreactions were visualized using an enhanced chemiluminescence kit (GE Healthcare, Chicago, IL, USA), in accordance with the manufacturer's protocol. The intensity of each band relative to $\beta$-actin was determined quantitatively using ImageQuant TL software (version 7.0; GE Healthcare Life Sciences). Data are reported as the mean \pm standard deviation of three replicates for each experiment.

Colony formation assay. HCC1937 cells were seeded in 6-well plates. Cells at $50 \%$ confluence were subsequently transfected with WT or mutant (T58A) MYC in triplicate and cultured for 12 days, followed by fixation with methanol and staining with $0.4 \%$ crystal violet at room temperature for $10 \mathrm{~min}$. Colonies containing $\geq 10$ cells were counted under an inverted microscope at magnification, $\mathrm{x} 100$. The colony formation ratio (\%) was determined as the number of cell clones divided by 500 and multiplied by 100 .

MTT assay. HCC1937 cells were transfected in 6-well plates and cultured for $48 \mathrm{~h}$. Cells with the indicated treatments were harvested and transferred to 96 -well plates. Following 24, 48 and $72 \mathrm{~h}$ incubation, the medium was removed and replaced with $100 \mu \mathrm{l}$ fresh culture medium. MTT $(5 \mathrm{mg} / \mathrm{ml}$; $20 \mu \mathrm{g}$ ) was added to each well, followed by incubation at $37^{\circ} \mathrm{C}$ for $4 \mathrm{~h}$. Dimethyl sulfoxide (100 $\mu \mathrm{g}$; Promega Corporation, Madison, WI, USA) was added to each well, followed by thorough mixing for $15 \mathrm{sec}$. Absorbance at $490 \mathrm{~nm}$ was measured using an automated plate reader. Each sample was analyzed in triplicate, and each experiment was repeated three times. Cell growth curves were calculated using mean values for each group.
TUNEL assay. Glass coverslips ( 30-mm diameter) were placed in the wells of a 6-well plate and cultured in RPMI-1640 medium. HCC1937 cells in the logarithmic growth phase were seeded in the plates at $1 \times 10^{5}$ cells/well. When the cells reached $50 \%$ confluence, they were transfected and cultured for $48 \mathrm{~h}$. Cell apoptosis was measured by the TUNEL assay according to the manufacturer's protocol. The cells were fixed in freshly prepared $4 \%$ methanol-free paraformaldehyde solution in PBS ( $\mathrm{pH} 7.4$ ) for $25 \mathrm{~min}$ at $4^{\circ} \mathrm{C}$. Following fixation, the cells were permeabilized in $0.2 \%$ Triton $\mathrm{X}-100$ solution in PBS for 5 min, washed with PBS and covered with $0.3 \% \mathrm{H}_{2} \mathrm{O}_{2}$ in PBS for $20 \mathrm{~min}$, at room temperature. The labeling reaction was performed using TUNEL reaction-mixture label solution incubated for $1 \mathrm{~h}$ at $37^{\circ} \mathrm{C}$. The samples were incubated with streptavidin-HRP for $30 \mathrm{~min}$ at room temperature. Following 3 PBS washes, the samples were stained with diaminobenzidine developing solution for $10 \mathrm{~min}$ at room temperature, and washed again 3 times. A total of 5 equal-sized fields were randomly chosen and analyzed under a Leica DMI 4000B light microscope (Leica Microsystems $\mathrm{GmbH}$ ). Density was evaluated in each positively stained field, yielding the density of dead cells (cell death index).

Annexin V-fluorescein isothiocyanate (FITC)/propidium iodide (PI) flow cytometry. Following $48 \mathrm{~h}$ of culture, cells in the indicated treatment groups were resuspended in binding buffer at a density of $1 \times 10^{6}$ cells $/ \mathrm{ml}$. A $100-\mu 1$ sample of cells was mixed with $5 \mu \mathrm{g}$ FITC-Annexin V (Sigma-Aldrich; Merck $\mathrm{KGaA})$ and PI (20 $\mu \mathrm{g} / \mathrm{ml}, 10 \mu \mathrm{l}$; Sigma-Aldrich; Merck KGaA). The cells were incubated for $20 \mathrm{~min}$ in the dark at room temperature, and $1 \times 10^{4}$ cells were analyzed from each sample by flow cytometry (FACSCaliber). Cell apoptosis was assayed using Cell Quest software (version 5.1; BD Biosciences). Cells that were positive for Annexin $\mathrm{V}\left(\right.$ Annexin $\mathrm{V}^{+}$) and negative for PI (PI') were scored as early apoptotic cells. Cells that were Annexin $\mathrm{V}^{+}$and $\mathrm{PI}^{+}$were scored as late apoptotic cells. In this way, necrotic cells were excluded (Annexin $\mathrm{V}^{+}$and $\mathrm{PI}^{-} / \mathrm{PI}^{+}$cells).

Statistical analysis. All statistical analyses were performed using SPSS 17.0 software (SPSS Inc., Chicago, IL, USA). Each result is presented as the mean \pm standard deviation of three replicate assays. One-way analysis of variance was used to analyze differences between groups. The LSD post hoc test was used to determine pairwise differences between means. $\mathrm{P}<0.05$ was considered to indicate a statistically significant difference.

\section{Results}

MYC mutant does not induce apoptosis in HCC1937 cells. HCC1937 cells were successfully transfected with WT and mutant MYC. Colony formation (Fig. 1A and B) and MTT (Fig. 1C) assays demonstrated that cell growth was induced by WT and mutant MYC compared with the control. Mutant MYC resulted in greater induction of cell proliferation compared with WT MYC.

TUNEL assays were performed to detect in situ apoptosis (Fig. 1D and E), with dark brown staining of nuclei indicating apoptotic cells. Compared with control cells, WT 
A

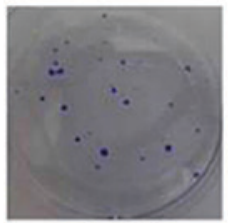

Control

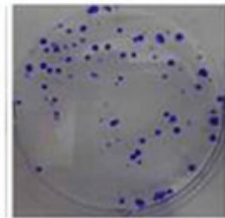

WT

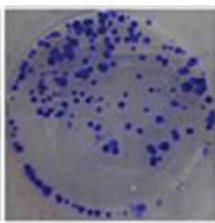

T58A
C

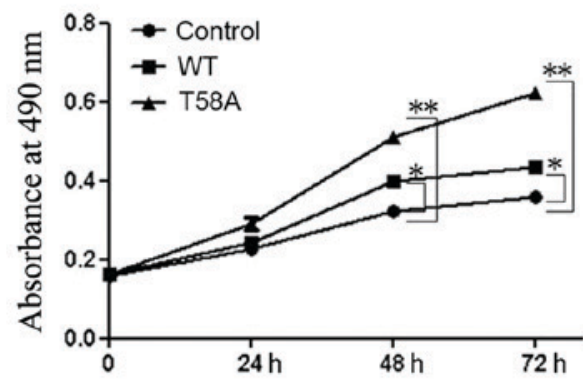

$\mathrm{E}$

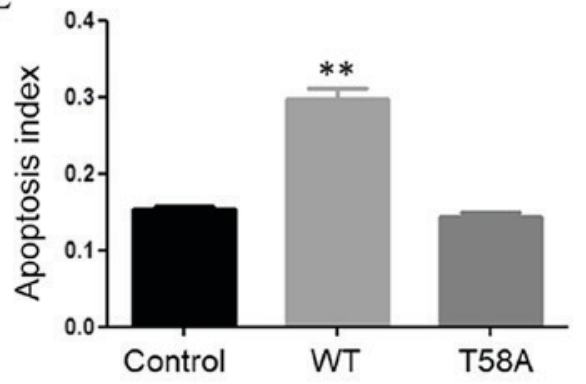

B

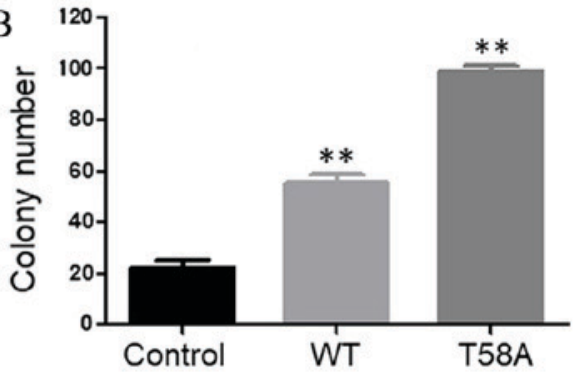

$\mathrm{D}$

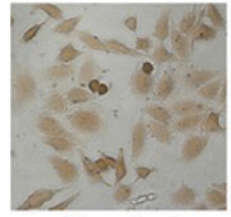

Control

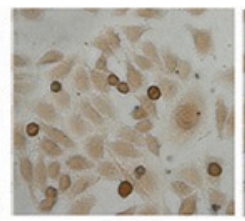

WT

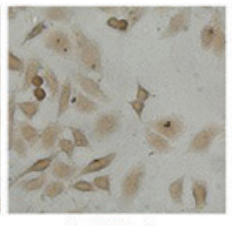

T58A
$\mathrm{F}$

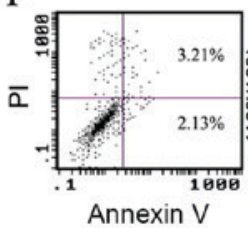

Control

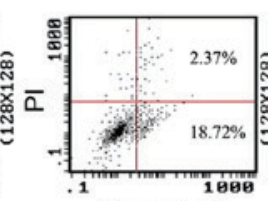

Annexin V

WT

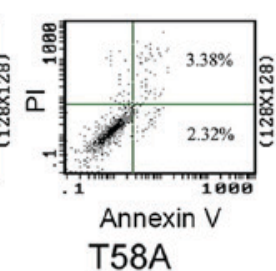

T58A

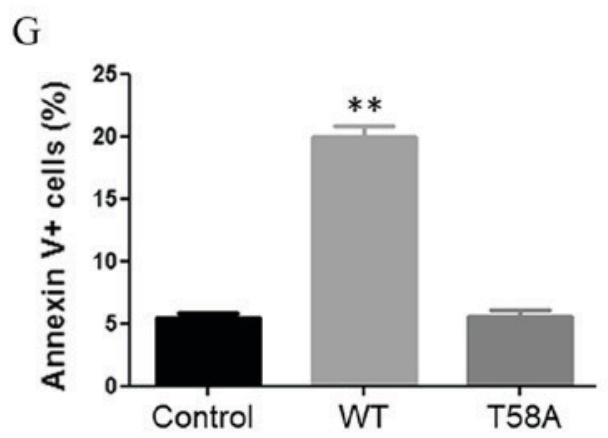

Figure 1. MYC mutant does not induce apoptosis in HCC1937 cells. (A) Colony formation observed by crystal violet staining of HCC1937 cells, following stable infection with control, WT MYC or T58A MYC vectors. (B) Colony number. (C) The MTT cell proliferation assay was performed 24-72 h following transfection. Absorbance at $490 \mathrm{~nm}$ was measured using an automated plate reader. The results were obtained from three replicate experiments. (D) Morphological alterations in HCC1937 cells were observed under an inverted microscope (magnification, x200). (E) Apoptosis rate of HCC1937 cells infected with control, WT MYC or T58A MYC vectors. (F) Cells were harvested and re-stained with Annexin V and PI, followed by flow cytometry to detect apoptosis. The relative number of cells (\%) that represents apoptosis is presented in each quadrant. (G) Apoptosis was determined as the percentage of Annexin $\mathrm{V}^{+}, \mathrm{PI}^{-} / \mathrm{PI}^{+}$cells. Each value represents the mean \pm standard deviation of three observations. ${ }^{*} \mathrm{P}<0.05$ and ${ }^{* *} \mathrm{P}<0.01$ vs. control. WT, wild-type; PI, propidium iodide.

cells displayed a higher apoptosis rate $(\mathrm{P}<0.01)$. No significant difference in apoptosis was observed between mutant MYC and control cells $(\mathrm{P}=0.30)$. Flow cytometry was performed in order to confirm the roles of mutant and WT MYC in apoptosis (Fig. 1F and G). The same results were obtained in the two experiments, indicating that mutant MYC induced proliferation and did not promote apoptosis in HCC1937 cells.

Impairment of Bim induced by mutant MYC. MYC was demonstrated to bind to Bim, which is involved in stimulus-induced p53-independent apoptosis (11-14). The expression levels of Bim in cells transfected with WT and mutant MYC were investigated (Fig. 2) to further examine why mutant MYC did not induce apoptosis. Compared with control cells, the Bim expression level was increased in WT cells $(\mathrm{P}<0.01)$. Bim expression was comparable in control and mutant MYC cells $(\mathrm{P}=0.92)$. These results indicated that the inability of mutant MYC to upregulate Bim is associated with its inability to induce apoptosis.

Mutant MYC is unable to suppress p21 or to induce Bim. The present study aimed to assess why mutant MYC is 

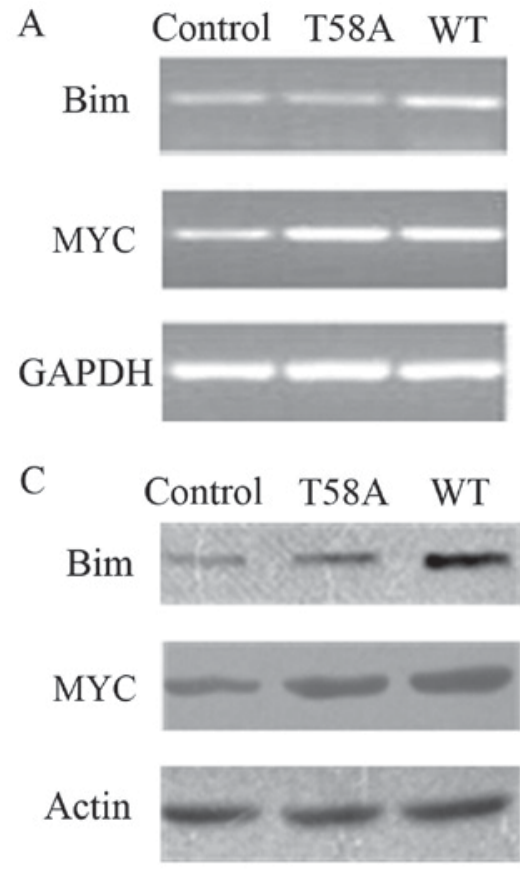

B
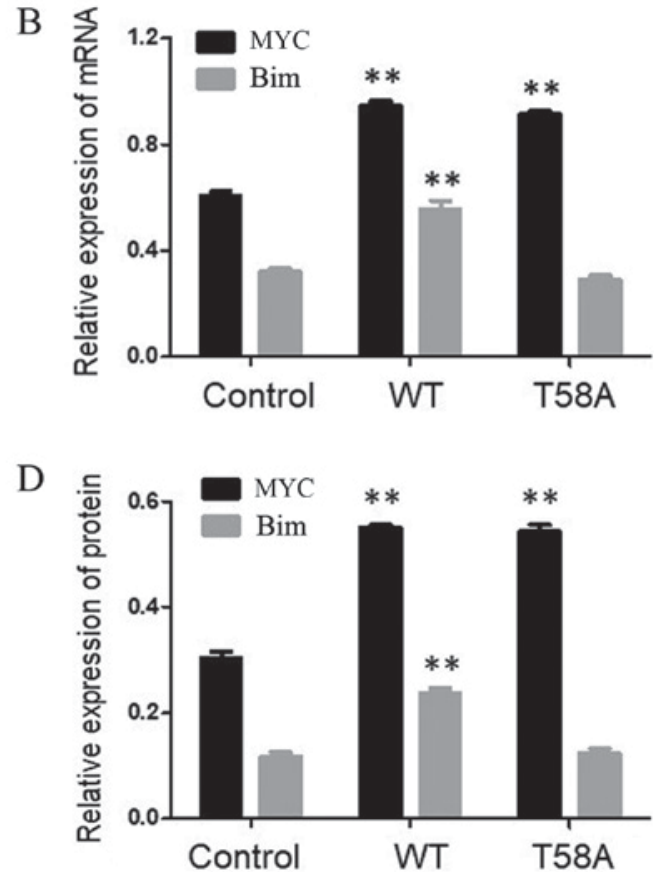

Figure 2. Expression levels of Bim in HCC1937 cells transfected with T58A and WT MYC. HCC1937 cells were stably infected with control, WT MYC or T58A MYC vectors. (A) Reverse transcription-semi-quantitative polymerase chain reaction analysis. (B) mRNA expression levels of MYC and Bim. (C) Western blot analysis. (D) Protein expression levels of MYC and Bim. GAPDH and $\beta$-actin were used as controls. Each value represents the mean \pm standard deviation of three observations. ${ }^{* * *} \mathrm{P}<0.01$ vs. respective control. WT, wild-type; MYC, Myc proto oncogene; si, small interfering.

unable to upregulate the expression of Bim (Fig. 3). Earlier studies supported the view that MYC represses the expression of p21 (31), while p21 represses the expression of Bim (23), thereby maintaining the balance between cellular proliferation and apoptosis. In the present study, in HCC1937 cells with p21 knockdown $(\mathrm{P}<0.01$; Fig. 3B and $\mathrm{E})$, the expression of Bim was increased $(\mathrm{P}<0.01$; Fig. $3 \mathrm{C}$ and $\mathrm{F})$. It was also demonstrated that p21 expression was significantly diminished $(\mathrm{P}<0.01$; Fig. 3B and E), and Bim expression was significantly increased $(\mathrm{P}<0.01$; Fig. 3C and F), in WT MYC-transfected cells compared with controls. In addition, when p21 was knocked down $(\mathrm{P}<0.01$; Fig. $3 \mathrm{~B}$ and $\mathrm{E})$, p21 expression was further diminished and Bim expression was further increased in WT MYC/p21-siRNA transfected cells compared with p21-siRNA transfected cells $(\mathrm{P}<0.01$; Fig. 3). These results suggested that $\mathrm{p} 21$ and Bim may be involved in a negative regulatory mechanism with MYC.

HCC1937 cells were transfected with mutant MYC and the expression levels of Bim and p21 were determined, using RT-sqPCR and western blot analyses (Fig. 3). Bim and $\mathrm{p} 21$ are associated with apoptosis and proliferation. The p21 and Bim expression levels were similar in mutant MYC and control cells ( $\mathrm{P}=0.85,0.82,0.51$ and 0.27 , respectively), indicating that mutant MYC was unable to suppress p21 or induce Bim. To confirm this regulatory mechanism, HCC1937 cells were transfected with T58A and the p21 siRNA construct (T58A/p21si). The expression level of p21 was demonstrated to be decreased ( $\mathrm{P}<0.01$; Fig. $3 \mathrm{~B}$ and $\mathrm{E})$, and the expression level of Bim was significantly increased $(\mathrm{P}<0.01$; Fig. 3C and F). This further demonstrated that mutant MYC was unable to suppress p21 or induce Bim. T58A MYC-transfected cells displayed marked proliferation, yet no induction of apoptosis $(\mathrm{P}<0.01$; Fig. 1$)$. In addition, these effects of mutant MYC on proliferation and apoptosis were weakened when cells were transfected with T58A and p21 siRNA together $(\mathrm{P}<0.05$; Fig. 4). These results indicated that the breast cancer-derived MYC mutation T58A does not suppress p21 or induce Bim, which may be the reason why it does not induce apoptosis.

Mutant MYC is unable to induce p14 or Bim. The tumor suppressor p14 is able to inhibit MYC-induced hyperproliferation and induce p53-independent apoptosis (18). In the present study it was reported that in HCC1937 cells transfected with p14 siRNA ( $\mathrm{P}<0.05$; Fig. 5), Bim expression was decreased $(\mathrm{P}<0.05$; Fig. 5C and F). WT cells displayed significantly increased expression levels of p14 and Bim (P<0.05; Fig. 5); however, when p14 was knocked down, p14 and Bim expression levels were further diminished in WT MYC/p14-siRNA transfected cells compared with p14-siRNA transfected cells $(\mathrm{P}<0.05, \mathrm{P}<0.01$; Fig. 5). Thus, p14 and Bim appear to form a positive regulatory mechanism with MYC.

To examine how mutant MYC regulated p14 and Bim, the expression levels of Bim and p14 were analyzed, using RT-sqPCR and western blot analyses in mutant MYC cells (Fig. 5). The expression levels of p14 and Bim were similar in mutant MYC and control cells $(\mathrm{P}=1.0,0.41,0.95$ and 0.25 , respectively). HCC1937 cells were transfected with T58A and the p14 siRNA construct (T58A/p14si). When p14 was blocked $(\mathrm{P}<0.05$; Fig. 5B and E), T58A/p14si cells displayed significantly lower expression levels of $\operatorname{Bim}(\mathrm{P}<0.01$; Fig. $5 \mathrm{C}$ and $\mathrm{F})$ compared with cells transfected with T58A alone. T58A MYC-transfected cells demonstrated notable proliferation and decreased apoptosis $(\mathrm{P}<0.01$; Fig. 1). In addition, these effects of mutant MYC on proliferation and apoptosis were strengthened when cells were transfected with T58A and the 
A

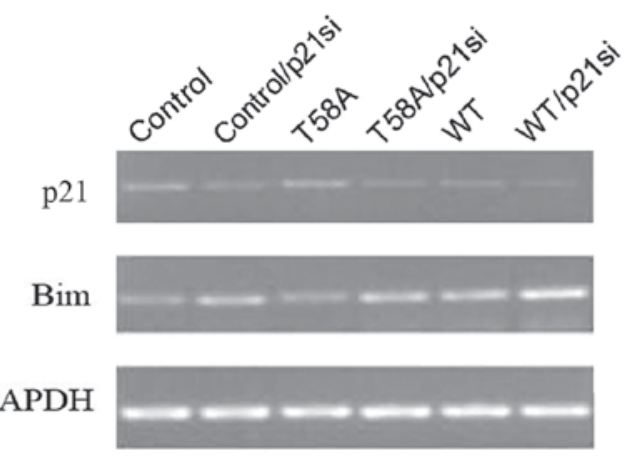

$\mathrm{C}$

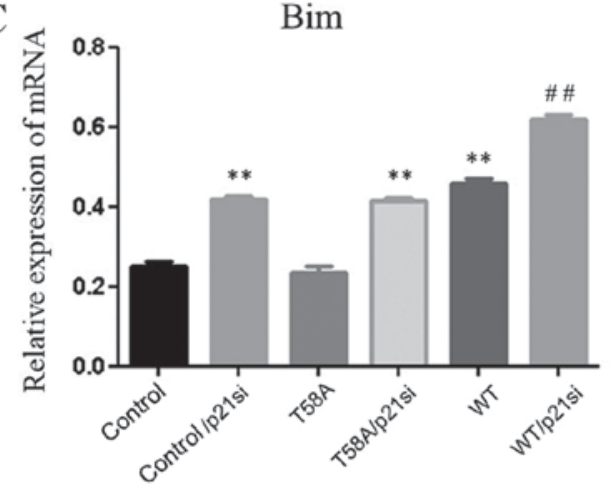

E

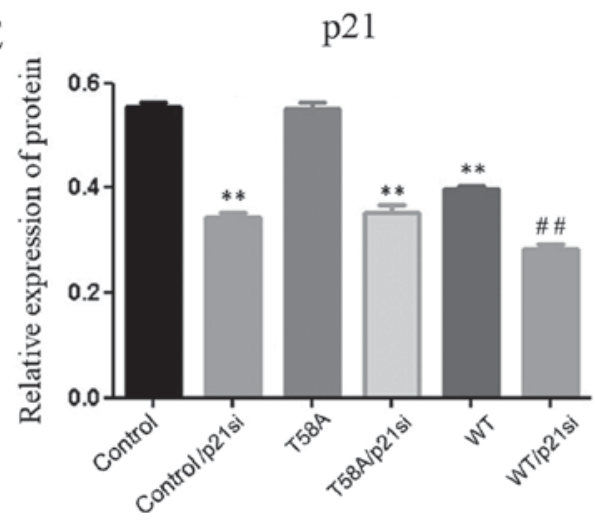

$\mathrm{B}$

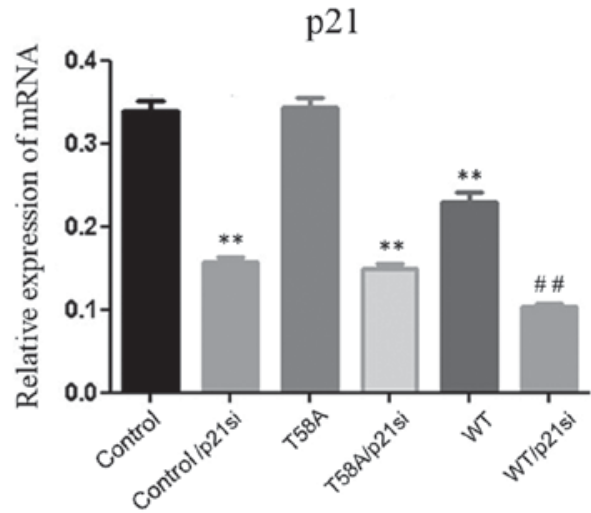

$\mathrm{D}$

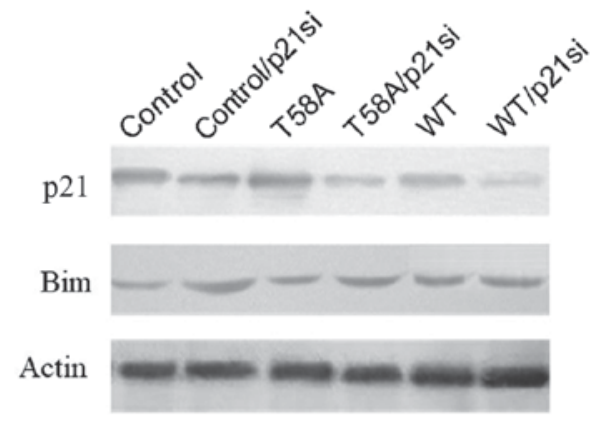

$\mathrm{F}$

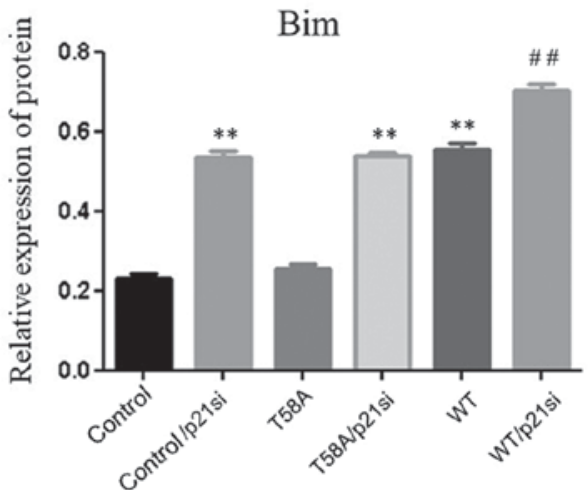

Figure 3. Mutant MYC does not suppress p21 or induce Bim. HCC1937 cells were stably infected with control, p21siRNA, WT MYC, WT and p21siRNA construct, T58A MYC vectors, and with T58A and p21 siRNA construct simultaneously. (A) Reverse transcription-semi-quantitative polymerase chain reaction analysis. (B) mRNA expression levels of p21. (C) mRNA expression levels of Bim. (D) Western blot analysis. (E) Protein expression levels of p21. (F) Protein expression levels of Bim. GAPDH and $\beta$-actin were used as controls. Each value represents the mean \pm standard deviation of three observations. ${ }^{* *} \mathrm{P}<0.01$ vs. control; ${ }^{\# \#} \mathrm{P}<0.01$ vs. control/p21si. WT, wild-type MYC; Myc proto-oncogene; si, small interfering.
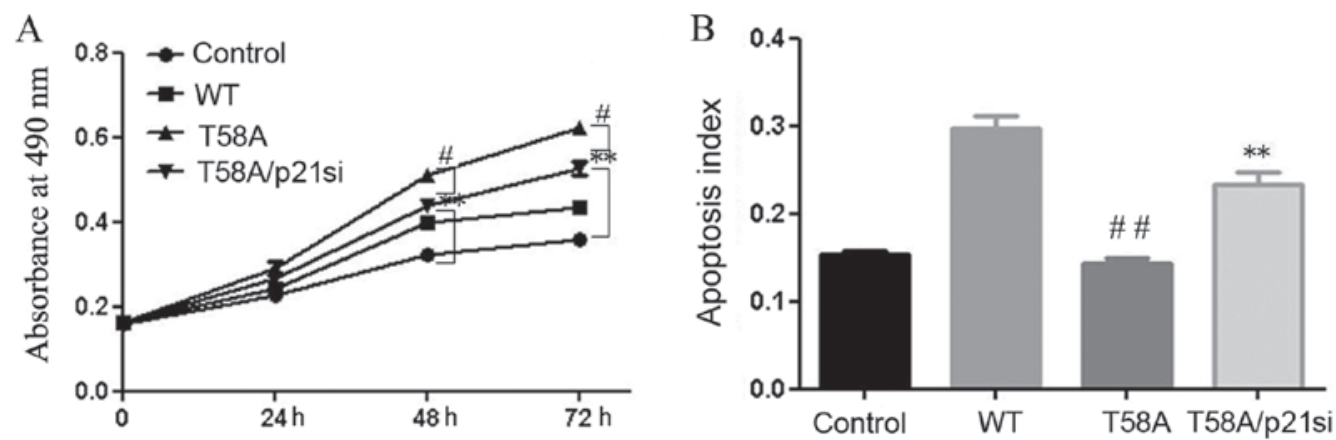

Figure 4. Effects of mutant MYC on proliferation and apoptosis are weakened when cells are co-transfected with T58A MYC and p21 siRNA. (A) MTT cell proliferation assay performed 24-72 $\mathrm{h}$ following transfection. Absorbance at $490 \mathrm{~nm}$ was measured with an automated plate reader. Results were obtained from three replicate experiments. (B) The terminal deoxynucleotidyl transferase-mediated dUTP nick end labeling assay detected the apoptosis rate of HCC1937 cells infected with control, WT MYC, T58A MYC vectors, and with T58A and the p21 siRNA construct at the same time (T58A/p21si). Each value represents the mean \pm standard deviation of three observations. ${ }^{* *} \mathrm{P}<0.01$ vs. control; ${ }^{*} \mathrm{P}<0.05$ and ${ }^{\# \#} \mathrm{P}<0.01 \mathrm{vs}$. T58A/p21si. WT, wild-type; si, small interfering. 
A

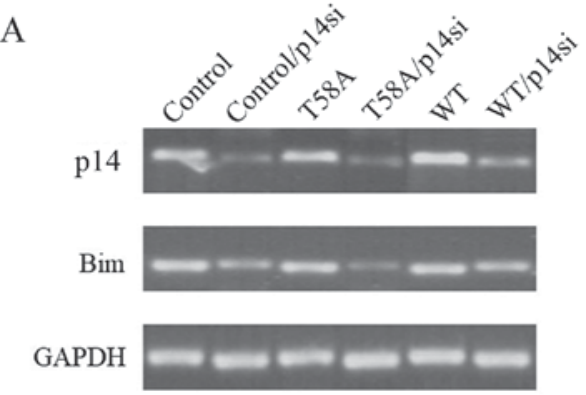

$\mathrm{C}$

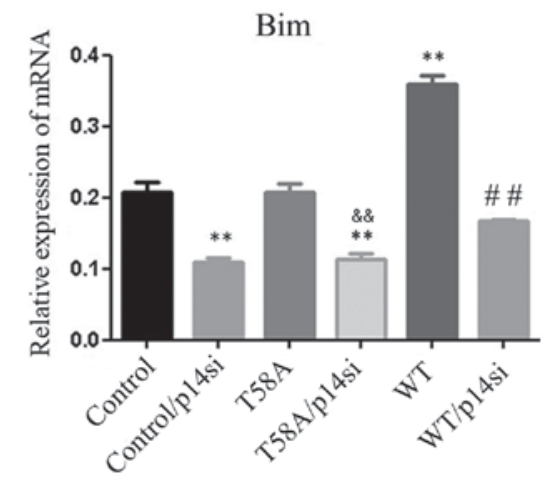

E

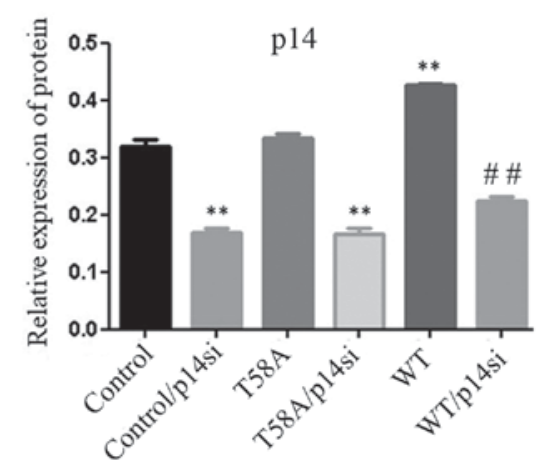

B

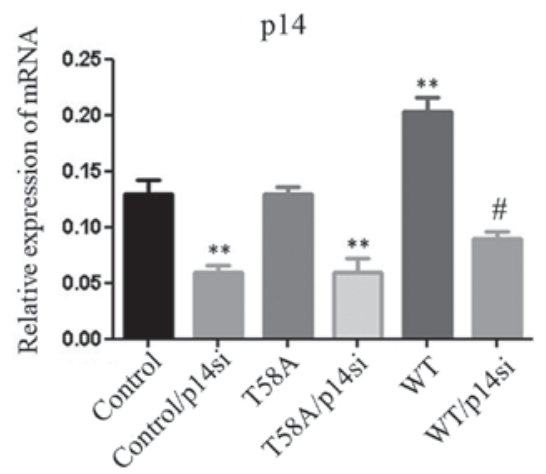

D

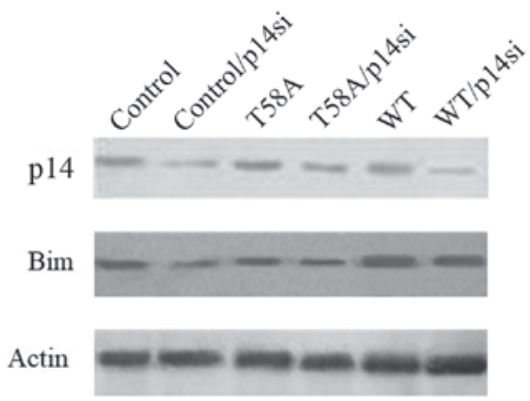

F

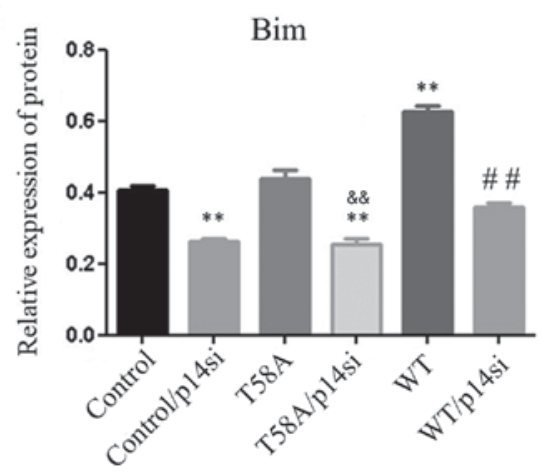

Figure 5. Mutant MYC does not induce p14 or Bim. HCC1937 cells were stably infected with control, p21siRNA, WT MYC, WT and p21siRNA construct, T58A MYC vectors, and with T58A and p14 siRNA construct at the same time. (A) Reverse transcription-semi-quantitative polymerase chain reaction analysis. (B) mRNA expression levels of p14. (C) mRNA expression levels of Bim. (D) Western blot analysis. (E) Protein expression levels of p14. (F) Protein expression levels of Bim. GAPDH and $\beta$-actin were used as controls. Each value represents the mean \pm standard deviation of three observations. ${ }^{* *} \mathrm{P}<0.01$ vs. control; ${ }^{\#} \mathrm{P}<0.05$ and ${ }^{\# \#} \mathrm{P}<0.01$ vs. control/p14si; \&\& $\mathrm{P}<0.01$ vs. T58A. WT, wild-type; si, small interfering.

p14 siRNA together $(\mathrm{P}<0.05$; Fig. 6). These results suggested that mutant MYC does not induce p14 or Bim, in contrast with previous reports. Hemann et al (9) reported that mutant MYC induced p14 and not Bim. This finding may have been due to the expression of p53. The results of the present study demonstrated that the breast cancer-derived MYC mutation T58A does not induce p14 or Bim, which may be another reason why it does not induce apoptosis.

\section{Discussion}

The results of the present study revealed novel insights into the biology of the MYC oncoprotein in breast cancer cells. In breast cancer with loss of p53, WT MYC and T58A mutant MYC are able to promote cell proliferation. In addition, WT MYC may also induce cell apoptosis, whereas T58A mutant MYC does not induce cell apoptosis. These results demonstrated that MYC, and not the MYC mutant, may be able to induce apoptosis in a p53-independent manner.
Hemann et al (9) previously reported that mutant MYC was unable to induce Bim expression, yet still activated the p53 pathway to a similar extent compared with WT MYC in Burkitt lymphoma. This may explain why tumors with mutant MYC are less prone to apoptosis (9). The results of the present study indicated that the tumor-derived MYC mutant T58A is relevant in breast cancer pathology, as it is unable to upregulate Bim or to induce apoptosis to protect against unrestrained proliferation in breast cancer.

Until now, MYC was thought to regulate Bim through the MYC-p14-Bim and MYC-p21-Bim networks, which interact with each other via mechanisms that are poorly understood. However, the reason why the MYC mutant is unable to promote apoptosis is unclear. Earlier studies supported the view that MYC represses the expression of p21, and that p21 represses the expression of Bim, to regulate cellular proliferation and the tumor surveillance response. In the present study, when HCC1937 cells were transfected with WT MYC, the expression level of p21 was significantly diminished and the 

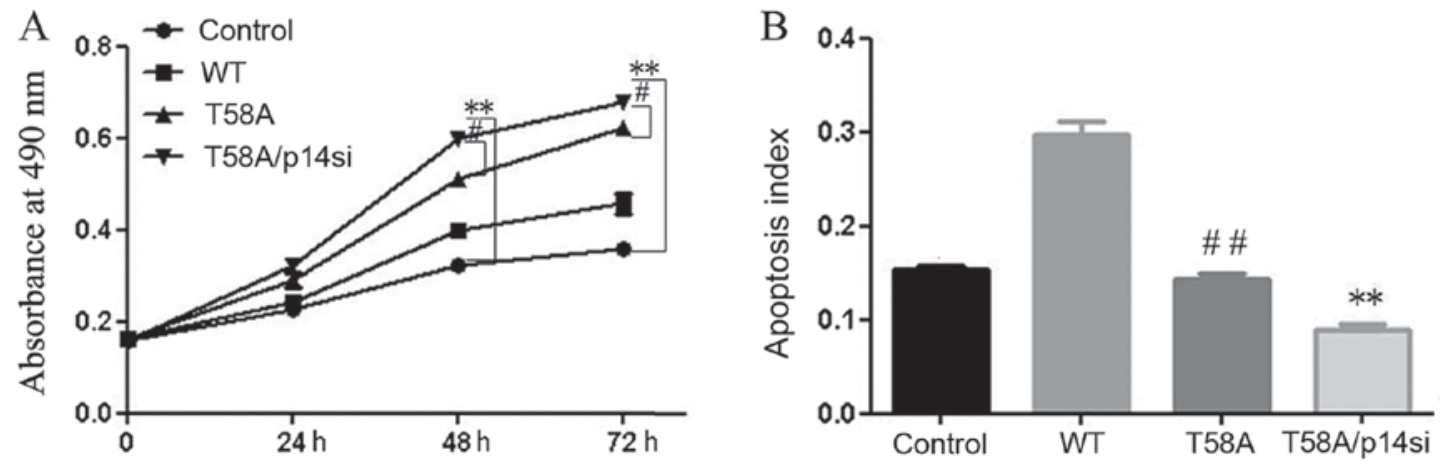

Figure 6. Effects of mutant MYC on proliferation and apoptosis are strengthened when cells are transfected with T58A and p14 siRNA simultaneously. (A) MTT cell proliferation assay performed $24-72 \mathrm{~h}$ following transfection. Absorbance at $490 \mathrm{~nm}$ was measured with an automated plate reader. Results were obtained from three replicate experiments. (B) The terminal deoxynucleotidyl transferase-mediated dUTP nick end labeling assay detected the apoptosis rate of HCC1937 cells infected with control, WT MYC, T58A MYC vectors, and with T58A and p14 siRNA construct simultaneously (T58A/p14si). Each value represents the mean \pm standard deviation of three observations. ${ }^{* *} \mathrm{P}<0.01$ vs. control; ${ }^{*} \mathrm{P}<0.05$ and ${ }^{\# \#} \mathrm{P}<0.01 \mathrm{vs}$. T58A/p14si. WT, wild-type; si, small interfering.

expression level of Bim was significantly increased. Thus, p21 and Bim seem to form a negative regulatory pathway with MYC. Furthermore, mutant MYC did not suppress p21, induce $\mathrm{Bim}$, or induce apoptosis to prevent unrestrained proliferation. When p21 was blocked, the expression of Bim was significantly increased and the effects of mutant MYC on proliferation and apoptosis were weakened. From these results, it was concluded that breast cancer-derived T58A MYC does not induce apoptosis, which is associated with its failure to suppress p21 and activate Bim.

p14 is a tumor suppressor protein, which is regulated by the MYC protein and is able to induce apoptosis (16). Further analysis has revealed that p14 may be combined with E2F to serve a role in tumor detection (19). The E2F family is a class of transcription factors that accumulate in Bim enhancers to regulate apoptosis induction. It seems that normal MYC increases p14, which interacts with Bim to induce apoptosis. The present study reported that the expression levels of p14 and Bim were significantly increased in WT MYC-transfected HCC1937 cells. These results confirm that p14 and Bim may form a positive regulatory association with MYC. It was also reported that mutant MYC was unable to upregulate p14 or Bim. When p14 was blocked, the expression level of Bim was significantly decreased due to transfected T58A. In addition, these effects of mutant MYC on proliferation and apoptosis were strengthened. Therefore, it was concluded that the breast cancer-derived MYC mutation T58A does not induce apoptosis, which is associated with its failure to activate p14 or Bim.

MYC-initiated alterations in gene expression have a variety of effects resulting in the formation of breast cancer. A previous study reported that the T58A mutation in MYC may enhance the formation of tumors (32). In recent work, the T58A mutations in MYC reduced the dependence on KRAS proto-oncogene mutations for tumorigenesis (33). In the present study, the T58A mutation was MYC is not able to regulate p14/p21 in MYC-induced apoptosis in p53-deficient breast cancer. However, the role of the T58A mutation in MYC-induced apoptosis is unclear. Further studies on the mechanism of the T58A MYC mutation in the development of breast cancer are required.

Substantial evidence has established that p53 is a key tumor suppressor, apoptosis-inducer and prognostic marker in cancer (34-36). The p53 status of a tumor may have a strong influence on tumor sensitivity to commonly used anticancer drugs and radiotherapy, given that the majority of anticancer treatments trigger DNA damage-induced apoptosis. The mechanism by which MYC activates Bim may provide important insights into novel tumor-specific treatment strategies, as activated Bim induces a p53-independent form of apoptosis that is induced by oncogenes rather than by DNA damage. Considering that p53 expression is lost in one-half of all human tumors (34), the unique MYC-p14-Bim and MYC-p21-Bim apoptosis pathways may have potential in the development of future therapeutic agents as an alternative to p53 reactivation.

\section{Acknowledgements}

The authors would like to thank Dr Xuefeng Zhang, Medical School of Qingdao University, Qingdao, China, for providing statistical advice, Professor Xiangping Liu, The Affiliated Hospital of Qingdao University (Qingdao, China), for technical support, and colleagues in the laboratory for helpful discussions.

\section{Funding}

The present study was funded by a grant from the Natural Science Foundation of Shandong Province (grant nos. y2007C134 and ZR2017PH032), the Higher Educational Science and Technology Program of Shandong Province (grant no. J17B092) and the National Natural Science Foundation of China (grant no. 81302290 and 81700029).

\section{Availability of data and materials}

The datasets used and/or analyzed during the current study are available from the corresponding author on reasonable request.

\section{Authors' contributions}

DJJ designed and performed experiments, and wrote the manuscript. YS and FL designed experiments and revised the manuscript. DJ helped to culture cells. WC and XW made 
substantial contributions to the conception and design of the study, and revised the manuscript. ZL and ZY helped to analyze the experimental data.

\section{Ethics approval and consent to participate}

Not applicable.

\section{Patient consent for publication}

Not applicable.

\section{Competing interests}

The authors declare that they have no competing interests.

\section{References}

1. Fallah Y, Brundage J, Allegakoen $\mathrm{P}$ and Shajahan-Haq AN MYC-Driven pathways in breast cancer subtypes. Biomolecules 7 : E53, 2017.

2. Eilers M and Eisenman RN: MYC's broad reach. Genes Dev 22: 2755-2766, 2008.

3. Meyer N and Penn LZ: Reflecting on 25 years with MYC. Nat Rev Cancer 8: 976-990, 2008.

4. Chen Y and Olopade OI: MYC in breast tumor progression. Expert Rev Anticancer Ther 8: 1689-1698, 2008.

5. Hynes NE and Stoelzle T: Key signalling nodes in mammary gland development and cancer: MYC. Breast Cancer Res 11: 210, 2009.

6. Efstratiadis A, Szabolcs M and Klinakis A: Notch, MYC and breast cancer. Cell Cycle 6: 418-429, 2007.

7. Chang DW, Claassen GF, Hann SR and Cole MD: The MYC transactivation domain is a direct modulator of apoptotic versus proliferative signals. Mol Cell Biol 20: 4309-4319, 2000.

8. Conzen SD, Gottlob K, Kandel ES, Khanduri P, Wagner AJ, O'Leary $\mathrm{M}$ and Hay $\mathrm{N}$ : Induction of cell cycle progression and acceleration of apoptosis are two separable functions of MYC: Transrepression correlates with acceleration of apoptosis. Mol Cell Biol 20: 6008-6018, 2000.

9. Hemann MT, Bric A, Teruya-Feldstein J, Herbst A, Nilsson JA, Cordon-Cardo C,Cleveland JL, Tansey WP and Lowe SW: Evasion of the p53 tumour surveillance network by tumour-derived MYC mutants. Nature 436: 807-811, 2005.

10. Thibodeaux CA, Liu X, Disbrow GL, Zhang Y, Rone JD, Haddad BR and Schlegel R: Immortalization and transformation of human mammary epithelial cells by a tumor-derived MYC mutant. Breast Cancer Res Treat 116: 281-294, 2009.

11. Delbridge AR, Pang SH, Vandenberg CJ, Grabow S, Aubrey BJ, Tai L, Herold MJ and Strasser A: RAG-induced DNA lesions activate proapoptotic BIM to suppress lymphomagenesis in p53-deficient mice. J Exp Med 213: 2039-2048, 2016.

12. Kovi RC, Paliwal S, Pande S and Grossman SR: An $\mathrm{ARF} / \mathrm{CtBP} 2$ complex regulates $\mathrm{BH} 3$-only gene expression and p53-independent apoptosis. Cell Death Differ 17: 513-521, 2010.

13. Muthalagu N, Junttila MR, Wiese KE, Wolf E, Morton J, Bauer B Evan GI, Eilers M and Murphy DJ: BIM is the primary mediator of MYC-induced apoptosis in multiple solid tissues. Cell Rep 8: $1347-1353,2014$

14. Campone M, Noël B, Couriaud C, Grau M, Guillemin Y, Gautier F, Gouraud W, Charbonnel C, Campion L, Jézéquel $\mathrm{P}$, et al: MYC dependent expression of pro-apoptotic Bim renders HER2-overexpressing breast cancer cells dependent on antiapoptotic Mcl-1. Mol Cancer 10: 110, 2011.

15. Liu X, Li F, Meng C, Jiang D and Liu S: Effects of mutant and wild-type c-myc on Bim expression in non-p53-dependent pathway. Med J Qilu 27: 395-397, 2012.
16. Datta A, Nag A, Pan W, Hay N, Gartel AL, Colamonici O, Mori Y and Raychaudhuri P: MYC-ARF (alternate reading frame) interaction inhibits the functions of MYC. J Biol Chem 279: 36698-36707, 2004.

17. Qi Y, Gregory MA, Li Z, Brousal JP, West K and Hann SR: p19ARF directly and differentially controls the functions of MYC independently of p53. Nature 431: 712-717, 2004.

18. Gregory MA, Qi Y and Hann SR: The ARF tumor suppressor: Keeping MYC on a leash. Cell Cycle 4: 249-252, 2005.

19. Aslanian A, Iaquinta PJ, Verona R and Lees JA: Repression of the Arf tumor suppressor by E2F3 is required for normal cell cycle kinetics. Genes Dev 18: 1413-1422, 2004.

20. Gogada R, Yadav N, Liu J, Tang S, Zhang D, Schneider A, Seshadri A, Sun L, Aldaz CM, Tang DG and Chandra D: Bim, a proapoptotic protein, up-regulated via transcription factor E2F1-dependent mechanism, functions as a prosurvival molecule in cancer. J Biol Chem 288: 368-381, 2013.

21. Abbas T and Dutta A: p21 in cancer: Intricate networks and multiple activities. Nat Rev Cancer 9: 400-414, 2009.

22. Dotto GP: p21WAF1/Cip1: More than a break to the cell cycle? Biochim Biophys Acta 1471: M43-M56, 2000.

23. Collins NL, Reginato MJ, Paulus JK, Sgroi DC, Labaer J and Brugge JS: G1/S Cell Cycle arrest provides anoikis resistance through erk-mediated bim suppression. Mol Cell Biol 25: 5282-5291, 2005.

24. Sherr CJ: The INK4a/ARF network in tumour suppression. Nat Rev Mol Cell Biol 2: 731-737, 2001.

25. Eischen CM, Roussel MF, Korsmeyer SJ and Cleveland JL: Bax loss impairs MYCinduced apoptosis and circumvents the selection of p53 mutations during MYCmediated lymphomagenesis. Mol Cell Biol 21: 7653-7662, 2001.

26. Soussi T and Wiman KG: Shaping genetic alterations in human cancer: The p53 mutation paradigm. Cancer Cell 12: 303-312, 2007.

27. Valente LJ, Gray DH, Michalak EM, Pinon-Hofbauer J, Egle A, Scott CL, Janic A and Strasser A: p53 efficiently suppresses tumor development in the complete absence of its cell-cycle inhibitory and proapoptotic effectors p21, Puma, and Noxa. Cell Rep 3: 1339-1345, 2013.

28. Strasser A, Harris AW, Jacks T and Cory S: DNA damage can induce apoptosis in proliferating lymphoid cells via p53-independent mechanisms inhibitable by Bcl-2. Cell 79: 329-339, 1994.

29. Huang DC and Strasser A: BH3-Only proteins-essential initiators of apoptotic cell death. Cell 103: 839-842, 2000

30. Meng CH, Li FN and Zhang DL: Construction and significance of lentiviral vector with c-myc. Chin J Exp Surg 4: 543-546, 2011.

31. Wanzel M, Herold S and Eilers M: Transcriptional repression by Myc. Trends Cell Biol 13: 146-150, 2003.

32. Wang X, Cunningham M, Zhang X, Tokarz S, Laraway B, Troxell M and Sears RC: Phosphorylation regulates c-Myc's oncogenic activity in the mammary gland. Cancer Res 71: 925-936, 2011.

33. Hollern DP, Yuwanita I and Andrechek ER: A mouse model with T58A mutations in Myc reduces the dependence on KRasmutations and has similarities to claudin-low human breast cancer. Oncogene 32: 1296-1304, 2013.

34. Farnebo M, Bykov VJ and Wiman KG: The p53 tumor suppressor: A master regulator of diverse cellular processes and therapeutic target in cancer. Biochem Biophys Res Commun 396: 85-89, 2010.

35. Mihara M, Erster S, Zaika A, Petrenko O, Chittenden T, Pancoska P and Moll UM: p53 has a direct apoptogenic role at the mitochondria. Mol Cell 11: 577-590, 2003.

36. Lee JY, Cho KS, Diaz RR, Choi YD and Choi HY: p53 expression as a prognostic factor in upper urinary tract urothelial carcinoma: A systematic review and meta-analysis. Urol Int 94: 50-57, 2015.

(i) $(2)$ This work is licensed under a Creative Commons Attribution-NonCommercial-NoDerivatives 4.0 International (CC BY-NC-ND 4.0) License. 